Meta

Journal des traducteurs

Translators' Journal

\title{
De la nécessité des enquêtes linguistiques pour le travail des commissions de terminologie
}

\section{Jean-Pierre Goudaillier}

Volume 32, numéro 3, septembre 1987

La fertilisation terminologique dans les langues romanes

URI : https://id.erudit.org/iderudit/002953ar

DOI : https://doi.org/10.7202/002953ar

Aller au sommaire du numéro

Éditeur(s)

Les Presses de l'Université de Montréal

ISSN

0026-0452 (imprimé)

1492-1421 (numérique)

Découvrir la revue

Citer cet article

Goudaillier, J.-P. (1987). De la nécessité des enquêtes linguistiques pour le travail des commissions de terminologie. Meta, 32(3), 361-365.

https://doi.org/10.7202/002953ar d'utilisation que vous pouvez consulter en ligne. 


\section{DE LA NÉCESSITÉ DES ENQUÊTES LINGUISTIQUES POUR LE TRAVAIL DES COMMISSIONS DE TERMINOLOGIE}

JEAN.PIERRE GOUDAILLIER

Université René Descartes, Paris, France

En matière de terminologie deux tendances coexistent. La première d'entre elles a pour but la mise en place d'une théorie de la néologie. Celle-ci doit permettre de forger des termes acceptables, c'est-à-dire non critiquables du point de vue de leur formation. Dans une telle perspective, la notion même d'usage réel du français n'a pas sa place et tenir compte du sentiment des locuteur(trice)s n'est en aucune manière envisagé. C'est sur ce point précis que le contraste est le plus marqué par rapport à l'autre tendance qui, quant à elle, accorde une grande importance non seulement aux comportements mais aussi aux attitudes des sujets parlants.

En tant que linguiste descriptiviste, la critique principale que je formule envers les listes de vocabulaire préconisées ces dernières années par les diverses commissions de terminologie ${ }^{1}$ porte sur le fait que l'usage n'ait pas été pris en ligne de compte et que des enquêtes linguistiques n'aient pas été effectuées lors de leur élaboration. Ces commissions ne sont d'ailleurs pas les seules à agir de la sorte, à négliger le sentiment linguistique des sujets parlants.

Si l'on prend en considération le seul domaine du remplacement de termes anglais ou franglais par des vocables français, la lecture des différentes listes de vocabulaire préconisées fait apparaître, par ailleurs, qu'il n'a nullement été tenu compte de la distinction entre un langage technique (jargons de spécialistes) et un langage commun; ainsi, le remplacement de teleprocessing par télétraitement d'une part, celui de cameraman par cadreur d'autre part ne peuvent pas être envisagés sur le même plan : caméraman fait partie du langage commun. Ce n'est pas le cas de télétraitement ou de teleprocessing.

Au hasard des listes j'ai pu noter des remplacements relevant d'incohérences, voire même d'erreurs importantes. En voici quelques exemples ${ }^{2}$ : ferry boat est déconseillé et remplacé par navire transbordeur ou transbordeur ${ }^{3}$. Comment penser que ce synthème ferry boat ou son abréviation ferry ne font pas partie du vocabulaire français ?

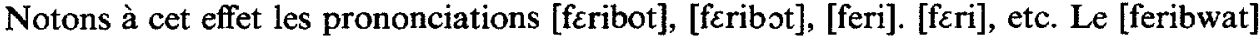
(*ferry $\sim$ boïte) de Marcel Pagnol l'a même bien ancré dans notre patrimoine littéraire et linguistique. Il n'est nullement évident que le sens de transbordeur soit perçu correctement par tous (cf. plus loin). Autre exemple : l'anglais - et franglais - pressurisation ne doit plus être utilisé, puisque l'équivalent mise en pression est conseillét. Par contre incrément, incrémenter, incrémentiel sont acceptés 5 . Or, l'anglais (te)increment existe. Pas d'" interdiction " cependant : increment serait-il ressenti comme " plus français " que pressurisation? Par qui, donc ? Quelle enquête linguistique le démontre ? Autre exemple du même type : derrick n'est pas conservé et tour (de forage) est préconisé pour le remplacer. Pipeline, sous réserve d'" être prononcé à la française ", est retenu ; de même pour management ${ }^{6}$. Pour quelles raisons derrick n'aurait-il pas pu être main- 
tenu, pour autant qu'il soit lui aussi prononcé à la française ? Ce qui est d'ailleurs le cas, lorsque l'on observe les prononciations de type [derik] rencontrées dans l'usage réel du français. À partir du moment où le signifiant d'un monème emprunté est traité conformément aux règles phonologiques, à celles de compositicn et/ou de dérivation de la langue opérant l'emprunt, quel critère objectif permet de considérer que ce terme n'est pas français? Dans bien des cas il n'est pas tenu compte du degré d'intégration dans la langue de certains emprunts : ce qui est le cas pour derrick; or, ce degré d'intégration est bien souvent la preuve du passage d'un terme dans le langage commun. Boulevard, mannequin sont de très bons exemples d'intégration phonologique du moyen néerlandais bolwero et du flamand mannekijn ${ }^{7}$. Attention, toutefois : l'intégration phonologique est une chose, l'intégration sémantique en est une autre. Les deux ne vont nullement de pair. Conséquence immédiate de la disjonction entre signifiant et signifié.

Le fait de préconiser le remplacement de tel ou tel terme franglais par un autre, ressenti et reconnu, quant à lui, comme "plus français", ne doit pas ressortir à la seule étude des moyens dont dispose le français pour "transposer " les mots issus de l'angloaméricain. Il ne suffit pas d'étudier les procédés de "transposition» des mots " étrangers ", de sélectionner ou de créer des mots nouveaux, encore faut-il procéder à l'analyse du degré d'intégration, tant sémantique que phonologique, voire même syntaxique, de tous ces termes et procéder aussi grâce à des enquêtes linguistiques portant non seulement sur les comportements mais aussi sur les attitudes des sujets parlants. Faute de quoi le produit que l'on souhaite promouvoir - le mot nouveau - pourrait se révéler n'être qu'un échec. Une simple analyse relative aux taux d'acceptabilité du nouveau terme peut le prévoir. C'est une des tâches du linguiste. Un exemple d'échec notable est fourni par bouteur, qui devait remplacer bulldozer ${ }^{8}$. L'enquête que j'ai faite ${ }^{9}$ montre que bouteur est inconnu pour $98 \%$ des enquêté(e)s, même si $94 \%$ d'entre eux(elles) indiquent que ce terme est français pour eux(elles). Bulldozer, quant à lui, est reconnu comme non français - anglais, dans le cas présent - par $94 \%$ des enquêté(e)s ; tous (100\%) utilisent bulldozer, qui plus est à bon escient. Transbordeur, préconisé en remplacement de ferry (boat), n'a guère eu de succès : seulement $35 \%$ des enquêté(e)s l'identifient en tant que bateau, alors que son équivalent franglais est connu à $91 \%$. Si cuisinette est relativement plus connu que ne l'est, par exemple, transbordeur $61 \%$ des enquêté(e)s le connaissent ${ }^{10}$-, son correspondant français l'est encore plus : à $81 \%$. Or, il est intéressant de noter que cuisinette est reconnu comme français à $100 \%$, kitchenette comme non français (anglais) à 100\% aussi ; le suffixe -ette de kitchenette ne suffit pas pour l'intégrer à la langue française, du moins dans la conscience des locuteur(trice)s. Ces quelques exemples montrent la complexité du problème et pour quelles raisons "l'intervention linguistique doit, pour être réussie, s'étayer d'une connaissance des usages et des attitudes (imaginaires) des sujets parlants... ", ainsi que le rappelle Anne-Marie Houdebine ${ }^{11}$.

L'exemple du français speaker et du québécois annonceur est particulièrement révélateur et indique la place prise par l'imaginaire linguistique ${ }^{12}$ dans ce que l'on désigne communément par conscience linguistique ou conscience des locuteur(e)s. Annonceur a un signifiant qui correspond aux règles de dérivation du français (annonc-er/annonceur). Speaker a une forme (son signifiant) ressentie comme anglaise. Il ne peut en être autrement. Si l'on demande à des Québécois quel est à leur avis le mot "le plus français ", leur réponse est invariablement : annonceur. Peut-on se satisfaire d'une telle réponse. Non! Annonceur masque des emplois anglais sous son signifiant français. Speaker, quant à lui, ne correspond à aucun des emplois faits en anglais de ce terme, puisqu'il est utilisé en français dans le sens de l'anglais announcer. Quel est donc "le plus français " des deux? Speaker? Annonceur? Le vocable québécois a un signifiant fran- 
çais, des emplois recouvrant ceux de l'anglais. Le terme français a un signifiant anglais, des emplois propres au français ... au franglais, plus précisément. Aucun critère objectif ne permet donc de dire que l'un est «plus français " que l'autre. Rien n'autorise à poser que le sémantique permet une meilleure intégration que le phonologique et vice versa. La réponse des Québécois montre par ailleurs qu'il s'agit de faire attention aux types de projections pouvant s'opérer à partir de sa propre langue : en effet, dans un tel cas, il a été accordé trop de crédit à la robe du mot et ses emplois en ont été alors oubliés. Préconiser le remplacement de speaker par annonceur en français revient donc à agir comme les Québécois et à proposer pour règle ses propres projections, qui ne relèvent, quant à elles, que du domaine du subjectif, de l'imaginaire linguistique. Qu'une commission de terminologie propose des termes essentiellement élaborés à partir des imaginaires linguistiques de ses membres ne peut aboutir dans bien des cas qu'à l'échec des innovations : celles-ci viendront souvent heurter les propres imaginaires des locuteur(trice)s et seront au départ d'une situation conflictuelle. Tenir compte des sujets parlants, de leurs usages par l'intermédiaire d'enquêtes peut permettre de promouvoir un produit meilleur - un terme accepté plus facilement - et d'éviter les échecs connus jusqu'à ce jour : à en croire Philippe Rossillon (séance d'ouverture du colloque), les diverses commissions de terminologie, qu'il a mises en place, ont proposé plus d'un millier de termes dont une bonne moitié n'a eu aucun avenir. Des enquêtes n'auraient-elles pas pu le prévoir et éviter une telle situation?

Une commission de terminologie a cependant essayé de travailler différemment : il s'agit de celle créée en février 1984 à propos de la féminisation des noms de métier ${ }^{13}$. Par ses méthodes de travail, cette commission se distingue des autres : des recherches d'attestations dans les dictionnaires, les textes officiels, etc., ainsi que des relevés de productions et d'attitudes auprès d'un grand nombre de locuteur(trice)s ont été effectués, avant même que des propositions ne soient émises pour donner lieu à un texte officiel en mars $1986^{14}$. À ce titre, elle me semble avoir été exemplaire, puisqu'il fallait bien qu'un travail en commission se fit, enquêtes linguistiques à l'appui, pour évaluer les chances de promotion de une professeur, une professeure, une professeuse, etc., d'une extinctrice face à une pompier, une pompière, etc., pour ne prendre que ces deux exemples en considération ${ }^{15}$. Apprendre que les plus jeunes classes d'âge privilégient l'utilisation d'une suivi d'un lexème épicène, que les locuteurs du sexe masculin, comme les enseignants, favorisent le masculin, ce qui est révélateur d'un conservatisme linguistique, est important et fournit une information indéniable au linguiste, mais aussi au décideur, au législateur. Pour auteur, marin, médecin, notaire, professeur, une théorie de la néologie retenant les seuls termes " acceptables et non critiquables" produirait les féminins autrice, marine ${ }^{16}$, médecine ${ }^{16}$, notairesse ${ }^{17}$, professeuse. Les enquêtes menées ont indiqué que les locuteurs retenaient une auteur, une marin, une médecin, une notaire, une professeur, autant d'épicènes précédés de l'article féminin. La prise de conscience de faits du type de ceux présentés ci-dessus a certainement contribué à la très grande prudence que l'on relève dans certaines formulations du document élaboré par la Commission; exemples parmi d'autres : " la Commission souhaiterait que la formation des féminins se fasse... ; consciente du fait que certaines formes risquent d'être mal acceptées, la Commission propose en premier lieu une forme épicène... ; entre ces différentes formes ... l'usage tranchera ». Le prescriptivisme outrancier est absent de telles formulations. Il ne s'agit pas de simples précautions oratoires ${ }^{18}$. Ainsi, sur ce point précis la Commission Benoîte Groult a-t-elle aussi été exemplaire. Première en France - souhaitons-le - d'un genre nouveau, qui permet d'opérer initialement d'aval en amont en tenant compte des usages linguistiques réels contemporains, voire même des desiderata des usagers d'une langue. Ce n'est que dans un deuxième temps que le mouvement peut être inversé, les décideurs 
répercutent d'amont en aval les mesures arrêtées ; en direction, donc, des sujets parlants eux-mêmes.

Grâce à cet exposé, je pense avoir montré qu'une concertation plus grande doit s'opérer entre décideurs et linguistes. Ne pas tenir compte des comportements et des attitudes des sujets parlants peut produire l'effet contraire de celui initialement recherché en étouffant toute initiative chez les usagers francophones français ou non. Les commissions de terminologie, à l'exception d'une seule (cf. plus haut), semblent avoir ceuvré jusqu'à présent malgré les usagers et non pas avec eux, pour eux, avec leur langue, pour leur langue ... pour notre langue. Il importe que les linguistes descriptivistes, entre autres, puissent dire leur mot en ce domaine. Je réitère ma mise en garde envers toute proposition qui émanerait des commissions, avant même que des enquêtes linguistiques à plus ou moins grande échelle n'aient pu établir le devenir de tel ou tel terme préconisé. Quelles sont les chances réelles de réussite de commanditaire pour sponsor, de parrainage pour sponsoring (cf. aussi à ce sujet la communication de Marie-Françoise Mortureux dans ce même colloque), de chalandage pour shopping, de prêt-à-manger pour fastfood $^{19}$ ? On peut certes se le demander. Une enquête nous fournirait des renseignements intéressants à ce sujet. Par ailleurs, s'il s'agit de trouver des mots qui soient en accord avec l'âme d'une langue, pour reprendre l'expression proposée par Philippe Rossillon ici-même, il convient qu'ils le soient aussi avec l'âme des sujets parlants ; faute de quoi, à l'aube du $21^{\mathrm{e}} \operatorname{siècle}^{20} \ldots$

Notes

1. Il s'agit des commissions instituées par le Décret $n^{\circ} 72-19 \mathrm{du} 7$ janvier 1972 relatif à l'enrichissement de la langue française ou par le Décret $n^{\circ} 83-243$ du 25 mars 1983 l'ayant abrogé et remplacé.

2. Cf. aussi Jean-Pierre Goudaillier (1977) : " $\dot{A}$ nouveau les puristes contre la langue", la Linguistique, 13-2, pp. 85-98, plus particulièrement pp. 95-98.

3. Arrêté du 12 janvier 1973 relatif à l'enrichissement du vocabulaire des transports.

4. Arrêté du 12 août 1974 relatif à l'enrichissement du vocabulaire en usage au ministère de la Défense.

5. Arrêtés des 22 décembre 1981 et 30 décembre 1983 relatifs à l'enrichissement du vocabulaire de l'informatique.

6. Pour derrick, tour (de forage) et pipeline, Arrêté du 12 janvier 1973 relatif à l'enrichissement du vocabulaire pétrolier ; pour management, Arrêté du 29 novembre 1973 relatif à la terminologie économique et financière.

7. Cf., entre autres, Albert Dauzat, Jean Dubois et Henri Mitterand (1964, éd. 1974) : Nouveau Dictionnaire étymologique et historique, pp. 102 et 442 ; voir aussi Jean-Pierre Goudaillier : "À nouveau... ", op. cit., pp. 89-90.

8. Arrêté du 12 janvier 1973 relatif à l'enrichissement du vocabulaire du bâtiment, des travaux publics et de l'urbanisme. Cet Arrêté a été abrogé par celui en date du 16 juillet 1984, et qui a été lui-même remplacé par l'Arrêté du 17 février 1986 relatif à l'enrichissement du vocabulaire de l'urbanisme et du logement.

9. Le questionnaire a été mis au point en 1977 et les passations de l'enquête ont eu lieu en 1978 et 1979. L'enquête a porté sur un total de 130 personnes, élèves d’École normale ou étudiant(e)s en lettres et/ou linguistique. Les résultats présentés ici concernaient 54 de ces 130 enquêté(e)s. Pour plus de détails, cf. JeanPierre Goudaillier (1982/1) : "Sprache und Macht : wie ein Gesetz in Frankreich die Sprache reinigen will ", Dialect, Wien, Schenel, pp. 28-51. Pour d'autres types de termes, voir Alice Cartier (1977) : "Connaissance et usage d'anglicismes par des Français de Paris ", la Linguistique, 13/2, pp. 57-83.

10. Le terme cuisinette est de toute manière identifié par sa forme, elle-même non ambiguë. On comprend tout de suite de quoi il s'agit, même si l'on ne connaît pas ce terme. Mais de là à l'utiliser ! Certain(e)s enquêté(e)s ont indiqué connaitre le terme sans pour autant l'employer eux(elles)-mêmes (des rubriques étaient prévues à cet effet par le questionnaire : connaissez-vous ce terme ? L'employez-vous ?) lui préférant kitchenette? 

NOTAISE, $\mathbf{J}$.

OVRUM, C.

PARC, F.

PAVAUT, $C$

PECHOUIN, D.

PETROFF, A.

PICO, $R$.

PICOCHE, J.

PIGAN, $C$.

PLASSARD, $F$.

POGACNIK, $v$.

PRADO, D.

QUEMADA, B.

RABILLAT, M.

RAIS, M.

RIBY, J.-Ph

SAGET, $L$.

SALAMA-CARR, $M$.

SANT, A.

SAVARY, $P$.

SCHAAR, $M$.

SERRANO, R.M.

SERVATIUS, $F$.

SIGAUD, B.

SLOUGUI, F.

SMITH, M.

SPIRE, M-L.

STONEHOUSE, $\mathrm{S}$.

STORZ, C.

SUOMELA-SALMI, E

SZTRUM, $M$.

TAZEROUALTI, $M$.

THOIRON, Ph.

TROJANUS, $\mathrm{K}$.-H.

TRUCHOT, $\mathrm{Cl}$.

VALETTE, F.

VICENTE LOZANO, J.-A

Van WILLIGEN, $M$.
Association des informaticiens de langue française

(France)

Université de Bergen (Norvège)

Groupe de terminologie du Canton de Berne (Suisse)

CNRS - INALF (France)

Librairie Larousse (France)

Université Paris X (France)

Université polytechnique de Catalogne

(Espagne)

Université d'Amiens (France)

Union latine (France)

Electricité de France (France)

Université de Ljubljana (Yougoslavie)

Union latine (France)

Institut national de la langue française -

INALF (France)

Ministère de l'Économie, des Finances et de la

Privatisation (France)

Centre de recherches statistiques économiques et sociales

et de formation pour les pays islamiques (Turquie)

Traducteur (France)

Ministère de la Défense (France)

Université de Salford (Grande-Bretagne)

Ministère de l'Économie, des Finances et de la

Privatisation (France)

Union technique de l'automobile (France)

INTRA EG (RFA)

Lycée François ter (France)

Ministère de l'Economie, des Finances et de la

Privatisation (France)

Traducteur (France)

Etudiante (France)

Thomson - CSF (France)

INRA (France)

Étudiante à Paris VII (France)

Etudiant à Paris III (France)

Etudiante à Paris V (France)

Union latine (France)

Étudiante (France)

Université de Lyon II (France)

OTAN (RFA)

Université de Strasbourg II (France)

DIXIT (France)

Lycée Jeanne d'Arc (France)

Université d'Utrecht (Pays-Pas) 
EISELE, $\mathrm{H}$.

ELUERD, $\mathbf{R}$.

ESQUIUS I. AUSIO, $R$.

ETTER, J.

FERNANDEZ,VEST, $J$.

FERRE, G.*A

FERRET CROS, $M$

FORTIN, J.M.

FREEDMAN, E.

FUNCK, J.E.

GALEAZZI, C.

GARSOU, $M$.

GEORGE, K.E.M.

GIGON, D.

GREAVES, A.

GREENBAUM, C

GRIGO, K.H.

HARRAS, G.

HEGELER-MAHE, E.

HENNING, J.-M

HIRSZA, $M$.

HUE-PARAREDA, $C$.

IBRAHIM

JEANDEL, A.

JOLIETTE, $\mathbf{C}$.

JUDGE, A

KANNAS, C.

KEDZIERSKA, $H$.

KHOUR Y, L.

KAECHLIN, J.-B.

LACASSE, $M$.

LANGE, $M$.

LE BALC'H, J.-Y.

IE BARS, A.

LEEUWIN-VAN DE VEN, A.

LEFEBVRE, $N$.

LÉTOURNEAU, D.

MASSON, $M$

MATEESCU, $H$

MATEU, $R$.

METAELIE, G.

MEVEL, J.-P.

MILLARD, $M$.

MOATI, $P$

MONEGO, J.

MOUNIN, $B$.

MONTEAU, M

MUNOZ CALVO, $M$.

NGUYEN DUY TAN

NICE, $R$.

NOAH-HAZOUME, A.
ISIT (France)

Université Sorbonne nouvelle (France)

Université polytechnique de Catalogne (Espagne)

Groupe de terminologie du Canton de Berne (Suisse)

CNRS - LACITO (France)

Commission nationale de terminologie de l'informatique (France)

Université polytechnique de Catalogne (Espagne)

Office de la langue française (Québec)

Institut français de gestion (France)

Traducteur (France)

IUT de Villetaneuse (France)

Service de la langue française (Belgique)

Université de Londres (Grande-Bretagne)

Groupe de terminologie du Canton de Berne (Suisse)

Université de Calgary (Canada)

Electricité de France - Gaz de France

Ruhrgas AG (RFA)

Institut für Deutschesprache (RFA)

Etudiante ESIT (France)

Université de Clermont II (France)

IUT de Saint-Denis (France)

Université de Saragosse (Espagne)

Université de Besançon (France)

Gaz de France (France)

Education nationale (France)

Université du Surrey (Grande-Bretagne)

Librairie Larousse (France)

CCIP (France)

Etudiante, Paris XIII (France)

CGEE ALSTHOM (France)

Office de la langue française (Québec)

Ministère de l'Économie, des Finances et de la

Privatisation (France)

Ministère de l'Environnement (France)

Université de Paris III (France)

Université d'Amsterdam (Pays-Bas)

CNRS - INALF (France)

Office de la langue française (Québec)

Université de Paris III (France)

AFNOR (France)

Institut de technologie de la construction de Catalunya (Espagne)

CNRS - Laboratoire d'ethnobotanique (France)

Librairie Larousse (France)

Ministère de l'Economie, des Finances et de la

Privatisation (France)

Ministère de l'Agriculture (France)

Université de l'Illinois (États-Unis)

Groupe de terminologie du Canton de Berne (Suisse)

Technicienne (France)

Collège universitaire de Téruel (Espagne)

Ministère de l'Economie, des Finances et de la

Privatisation (France)

Université de Surrey (Grande-Bretagne)

Société nationale des eaux du Cameroun 


\author{
MEUNIER-CRESPO, M. \\ MORTUREUX, M.F. \\ NGANDU, $P$. \\ PETIOT, G. \\ PORTELANCE, C. \\ PREVOS, A. \\ ROSSILLON, Ph. \\ SABIN, $S$. \\ SANTOYO, J.-C. \\ SCHAPIRA, C. \\ STAMMERJOHANN, $\mathrm{H}$. \\ SYPNICKI, J. \\ TRESCASES, $P$ \\ VAN DETH, J.-P. \\ VIRGILIO (di), $\mathbf{P}$ \\ ZWANENBURG, $N$
}

RAPPORTEURS

ALGARDY, $F$

MOSLER, A.-M.

BERMAN, A.

\section{LISTES DES PARTICIPANTS}

ALTHOFF, $E$.

AMIRA, A.

ANDRIEUX, $C$

ARIOTTI, A.

ATELLIAN, $\boldsymbol{J}$.

BARADUC, J.

BECHEREL, D.

BECQUER, A.-M

BÉJOINT, $H$

BERNAT, G.

BEYEL, $D$.

BIDARD, $\mathbf{R}$.

BOISSEAU, $Y$.

BOSWELL, $C$.

BOTTON, J.

BRELY, $L$.

CAMBOURS, $\mathrm{C}$.

CAMPOS, $G$

CANCEILL, B.

CANCEILL, $M$

CARRIOT, $\mathrm{C}$.

CHERIGUEN, $F$

CHETOUANI, L.

CHOLETTE, M.

CINTRAT, I.

CORBEIL, J.-C.

COUDERT, $P$.

COUTIER, $M$.

DABROWSKA, $J$

DAVID, J.

DAX (de), $F$.

DONZEL, $F$.

DREVET, E.
Université de Lyon III (France)

Université de Paris $X$ (France)

Université de Annaba (Algérie)

Université de Paris III (France)

Université de Montréal (Québec)

Pennsylvania State University (États-Unis)

Union latine (France)

Université de Francfort (Allemagne)

Université de León (Espagne)

TECHNION d'Haifa (Israël)

Université de Francfort (Allemagne)

Université de Poznan (Pologne)

Université de Fredericton (Canada)

FRANTERM (France)

Université de Toronto (Canada)

Université d'Utrecht (Pays-Bas)

\author{
FRANTERM (France) \\ CIREEL (France) \\ CIREEL (France)
}

Traductrice (France)

Etudiant (Belgique)

Commissariat à l'Énergie atomique (France)

Ingénieur civil des mines (France)

CEDRE (France)

Université d'Orléans (France)

Université de Brest (France)

CNRS - INALF (France)

Université de Lyon II (France)

TEKELEC-AIRTRONIC (France)

INTRA EG (RFA)

Cercle Blaise-Pascal (France)

Librairie Larousse (France)

Université de Londres (Grande-Bretagne)

Traductrice (France)

le Quotidien du médecin (France)

AFNOR (France)

Cie des experts traducteurs et interprètes

de la Cour d'appel de Paris (France)

CNET (France)

AUPIC (France)

Direction des monnaies et médailles (France)

Université d'Alger (Algérie)

Etudiante, Paris XIII (France)

Office de la langue française (Québec)

CREDIF (France)

CIRELFA (Québec)

Traductrice (France)

CNRS - INALF (France)

Écrivain public (France)

Comité de normalisation et de soudure (France)

ISIT (France)

COFORMA (France)

INRA (France) 


\section{LA FERTILISATION TERMINOLOGIQUE PARIS, 24-25 OCTOBRE 1986}

COMITÉ D'HONNEUR

BEHAR, $\mathrm{H}$.

DETH. J.-P. (van)

GERBOD, $P$.

JAISSON, $P$.

MARTIN, R

PERROT, J.

POTTIER, $B$

QUEMADA, B.

REY, A.

SELESKOVITCH, D.

SOURIOUX, J.-C

\section{COMITÉ SCIENTIFIQUE}

CHARAUDEAU, $P$

CHAURAND, J.

CLING, M.

HOUDEBINE, A.-M.

LAROCHE-BOUVY, D.

MITTERAND, $H$

ROUDIL, J.

\section{COMITÉ D'ORGANISATION}

ALGARDY, $F$

BESSE (de), B.

LEDUC-ADINE, J.-L.

LERAT, $P$.

MOSLER, A.M.

\section{PRÉSIDENTS DE SÉANCE}

AUGER, $P$.

CUMMINS, P.W.

DURAND, A

EICHFELDER, D.

HANSE, J.

JAISSON, $P$.

KAROLAK, $S$

QUEMADA, B.

ROUDIL, J.

WELLS, W.

DEPECKER, L.

\section{INTER VENANTS}

AZEVEDO-FERREIRA (de), $J$.

BESZERDA, 1 .

BOULANGER, J.-Cl.

CAPUTO, A.

CLAS, A.

DON, D.

ENRICO, E

GAMBIER, $Y$

GOUDAILLIER, J.-P.

HUMBLEY, J.

JASTRAB, M.J.

LENOBLE-PINSON, M
Université de Paris III (France)

FRANTERM (France)

Université de Paris XIII (France)

Université de Paris XIII (France)

Université de Paris IV (France)

Université de Paris III (France)

Universitê de Paris IV (France)

CNRS - INALF (France)

Dictionnaires le Robert (France)

ESIT (France)

Université de Paris II (France)

Université de Paris XIII (France) Université de Paris XIII (France)

Universitć de Paris XIII (France)

Université d'Angers (France)

Université de Paris III (France)

Université de Paris III (France)

Université de Paris XIII (France)

\section{FRANTERM (France)}

ESIT (France)

Université de Paris HI (France)

Université de Paris XIII (France)

CIREEL (France)

(Office de la langue française (Québec)

Northern Arizona University (États-unis)

AFNOR (France)

UNESCO (France)

Conseil de la langue française (Belgique)

Université de Paris XIII (France)

École normale supérieure de Cracovie (Pologne)

Institut national de la langue française (France)

Université de Paris XIII (France)

Secrétariat d'Etat (Canada)

Commissariat général de la langue française (France)

Université de Minho (Portugal)

Université de Poznan (Pologne)

Université Laval (Québec)

Université de Rome (Italie)

Université de Montréal (Québec)

FRANTERM (France)

Université de Rome (Italie)

Université de Turku (Finlande)

Université de Paris V (France)

Université de Nancy II (France)

Paris (France)

Facultés universitaires Saint-Louis (Belgique) 
11. Anne-Marie Houdebine (1983) : "Problématique d'une recherche", Médias \& langage, 19-20, pp. 61-63, p. 61.

12. Pour ce concept, cf. Anne-Marie Houdebine (1985) : "Pour une linguistique synchronique dynamique ", la Linguistique, 21, pp. 7-36.

13. Décret $n^{\circ} 84-153$ du 29 février 1984 portant création de la commission de terminologie relative au vocabulaire concernant les activités des femmes.

14. Circulaire du 11 mars 1986 relative à la féminisation des noms de métier, fonction, grade ou titre.

15. Anne-Marie Houdebine : "Problématique... ", op. cit., pp. 62 et 63 ; voir aussi Régine Luongo (1985) : Dynamique lexicale : la féminisation des noms de métier, mémoire de maîtrise en sciences du langage, Université d'Angers/Université Paris III.

16. Inutile d'insister - me semble-t-il — sur les risques de collision de marine et médecine avec des termes existant déjà dans la langue. Femme-marin et femme-médecin sont les usages québécois correspondant respectivement à une marin et une médecin.

17. Tout dérivé en -esse est difficilement accepté, ce que révèlent les enquêtes.

18. De telles formulations qui se trouvaient dans le texte proposé par la Commission pour donner lieu à un Arrêté (cf. Dossier de presse relatif à la Commission Groult) ne se retrouvent pas dans le texte définitif de la Circulaire du 11 mars 1986 (cf. note 14 plus haut), ce à cause de leur caractère flou, incompatible avec la rigueur exigée de tout texte législatif.

19. Arrêté du 17 mars 1982 relatif à l'enrichissement du vocabulaire du tourisme. Pour chalandage, ne pas oublier magasinage du québécois, cité d'ailleurs par l'Arrêté en tant que québécisme.

20. Une autre version de ce texte - plus étendue sur certains points - doit paraître dans Dilbilim (revue du Département de français de la faculté des lettres de l'Université d'Istambul) sous le titre : « Pour ou contre la langue : vingt ans de planification linguistique. »

RÉFÉRENCES BIBLIOGRAPHIQUES

CARTIER, Alice (1977) : " Connaissance et usage d'anglicismes par des Français de Paris ", la Linguistique, $13 / 2$, pp. 55.83 .

DAUZAT, Albert, Jean DUBOIS \& Henri MITTERAND (1964, éd. 1974) : Nouveau dictionnaire étymologique et historique, Paris, Larousse, $804 \mathrm{p}$.

GOUDAILLIER, Jean-Pierre (1977) : "À nouveau les puristes contre la langue ", la Linguistique, 13/2, pp. 85-98.

GOUDAILLIER, Jean-Pierre (1982) : "Sprache und Macht : wie ein Gesetz in Frankreich die Sprache reinigen will", Dialect, Wien, Schendl, pp. 28-51.

GOUDAILLIER, Jean-Pierre (à paraître) : « Pour ou contre la langue : vingt ans de planification linguistique en France", Dilbilim.

HOUDEBINE, Anne-Marie (1983) : "Problématique d'une recherche linguistique ", Médias \& langage, 19-20, pp. 61-63.

HOUDEBINE, Anne-Marie (1985) : «Pour une linguistique synchronique dynamique », la Linguistique, 21, pp. 7-36.

LUONGO, Régine (1985) : Dynamique lexicale : la féminisation des noms de métiers, mémoire de maîtrise en sciences du langage, Université d'Anvers/Université Paris III. 